\title{
Validation of Self-administrated Questionnaire for Psychiatric Disorders in Patients with Functional Dyspepsia
}

\author{
Ada W.Y. Tse, M.D. ${ }^{1}$, Larry H. Lai, M.D. ${ }^{2}$, C.C. Lee, M.D. ${ }^{1}$, Kelvin K.F. Tsoi, Ph.D. ${ }^{2}$, Vincent W.S. Wong, M.D. ${ }^{2}$, Yawen Chan \\ M.Sc. ${ }^{2}$, Joseph J.Y. Sung, M.D., Ph.D. ${ }^{2}$, Francis K.L. Chan, M.D. ${ }^{2}$, and Justin C.Y. Wu, M.D. ${ }^{2 *}$ \\ ${ }^{1}$ Kwai Chung Hospital, Hospital Authority, Hong Kong S. A. R., China, ${ }^{2}$ Institute of Digestive Disease, The Chinese University of Hong Kong \\ S.A.R., China
}

\section{Introduction}

Psychiatric comorbidity is common in patients with functional dyspepsia (FD) but a good screening tool for psychiatric disorders in gastrointestinal clinical practice is lacking.

Aims

1) Evaluate the performance and optimal cut-off of 12-item General Health Questionnaire (GHQ-12) as a screening tool for psychiatric disorders in FD patients; 2) Compare health-related quality of life (HRQoL) in FD patients with and without psychiatric comorbidities.

\section{Methods}

Consecutive patients fulfilling Rome III criteria for FD without medical co-morbidities and gastroesophageal reflux disease were recruited in a gastroenterology clinic. The followings were conducted at 4 weeks after index oesophagogastroduodenoscopy: self-administrated questionnaires on socio-demographics, dyspeptic symptom severity (4-point Likert scale), GHQ-12, and 36-item short-form health survey (SF-36). Psychiatric disorders were diagnosed with Structured Clinical Interview for DSM-IV Axis I Disorders (SCID) by a trained psychiatrist, which served as reference standard.

\section{Results}

55 patients underwent psychiatrist-conducted interview and questionnaire assessment. 27 (49.1\%) had current psychiatric disorders as determined by SCID (anxiety disorders: 38.2\%, depressive disorders: $16.4 \%$ ). Receiver operating characteristic curve analysis of GHQ-12 revealed an area under curve of $0.825(95 \% \mathrm{Cl}: 0.698-0.914)$. Cut-off of $\mathrm{GHQ}-12$ at $\geq 3$ gave a sensitivity of $63.0 \%(95 \% \mathrm{Cl}=42.4-80.6 \%)$ and specificity of $92.9 \%(95 \% \mathrm{Cl}=76.5 \%-98.9 \%)$. Subjects with co-existing psychiatric disorders scored significantly lower in multiple domains of SF-36 (mental component summary, general health, vitality and mental health). By multivariate linear regression analysis, current psychiatric morbidities (Beta $=-0.396, p=0.002$ ) and family history of psychiatric illness (Beta $=-0.299, p=0.015$ ) were independent risk factors for poorer mental component summary in SF-36, while dyspepsia severity was the only independent risk factor for poorer physical component summary (Beta $=-0.332, p=0.027$ ).

\section{Conclusions}

Concomitant psychiatric disorders adversely affect HRQoL in FD patients. The use of GHQ-12 as a reliable screening tool for psychiatric disorders allows early intervention and may improve clinical outcomes of these patients.

(J Neurogastroenterol Motil 2010;16:52-60)

Key Words

Dyspepsia, Questionnaires, Psychiatric diagnosis, Quality of life

Received: November 6th, 2009 Accepted: January 5th, 2010

(c) This is an Open Access article distributed under the terms of the Creative Commons Attribution Non-Commercial License (http://creativecommons. org/licenses/by-nc/3.0) which permits unrestricted non-commercial use, distribution, and reproduction in any medium, provided the original work is properly cited.

*Correspondence: Justin C.Y. Wu, M.D., FHKAM

Department of Medicine and Therapeutics, 9th Floor, Clinical Science Building, Prince of Wales Hospital, 30-32 Ngai Shing Street, Shatin, Hong Kong S.A.R., China

Tel: +852-2632-3593, Fax: +852-2637-3852, E-mail: justinwu@cuhk.edu.hk

Financial support: This study was supported by research fund of Department of Medicine \& Therapeutics and Li Ka Shing Insitute of Health Sciences, The Chinese University of Hong Kong.

Conflicts of interest: None. 


\section{Introduction}

Functional dyspepsia (FD) has been strongly associated with psychiatric disorders. It has been reported that $34 \%$ of FD patients had psychiatric disorders based on semi-structured comprehensive psychopathological rating scale, as compared with $15 \%$ and $1 \%$ in duodenal ulcer patients and healthy control, respectively. ${ }^{1} \mathrm{FD}$ patients have also been demonstrated to have higher anxiety and depressive scores compared to non-dyspeptic controls. $^{2-4}$ Owing to the lack of expertise in diagnosis and management, depressive and anxiety disorders are often left undiagnosed or untreated among FD patients in both primary and specialist settings, which leads to poor psychiatric as well as medical outcomes. Despite the extensive use of various screening questionnaires, few studies applied the gold standard psychiatrist-conducted diagnostic interview to confirm these conditions and the validity of these screening instruments in FD patients remained unclear. It has been generally accepted that the 12-item General Health Questionnaire (GHQ-12) is a useful tool for psychological disorders in general practice and community setting and it has been translated into sixteen different languages. ${ }^{5,6}$ Nevertheless, its optimal cut-off values, sensitivities and specificities vary significantly across different medical conditions and different settings, ${ }^{6}$ and not to mention the absence of data for its utility in FD. Hence, it is necessary to evaluate its performance and identify the optimal cut-off before routine usage in FD patients.

Health-related quality of life (HRQoL) is now believed to be the key indicator for the burden of chronic disease, including FD. ${ }^{7-12}$ Significant reduction in both physical and mental domains of 36-item short-form health survey (SF-36) has been demonstrated in FD patients. ${ }^{11}$ However, few studies investigate the impact of psychiatric morbidities on HRQoL of FD patients.

We set out to conduct a prospective study to validate the GHQ-12 as a screening instrument for psychiatric disorders in FD patients, using Structured Clinical Interview for DSM-IV Axis I Disorders (SCID) as a reference standard. We also compared the SF-36 scores between FD patients with and without psychiatric disorders.

\section{Materials and Methods}

This was a prospective case series conducted in the gastroenterology clinic of Prince of Wales Hospital, Hong Kong. Consecutive patients who were newly referred from primary care clinicians for dyspepsia were screened. Upper endoscopy was arranged within 2 weeks after the initial visit. Those patients who had normal endoscopy examination were deemed eligible.

These patients were then invited to complete the Rome III dyspepsia module questionnaire. ${ }^{13}$ All patients over 65 years old were also screened by the Cantonese version of Mini-mental State Exam (MMSE), and those with cognitive impairment as defined by MMSE score below respective cut-off were excluded (18 for illiterate subjects, 20 for those with 1 to 2 years of schooling, and 22 for those with more than 2 years of schooling). ${ }^{14}$ The inclusion criteria included (1) Rome III diagnostic criteria for FD, ${ }^{15}$ (2) Chinese ethnicity, and (3) age 18 years or above. Patients with significant medical conditions (any organic physical condition that required regular follow up or treatment e.g., hypertension, diabetes mellitus), predominant reflux symptoms, illiteracy, suspected dementia, failure to give consent, and pregnancy were excluded. Written informed consent would be obtained after verification of inclusion and exclusion criteria at the index endoscopy. Ethics approval was obtained from Joint Chinese University of Hong Kong - New Territories East Cluster Clinical Research Ethics Committee.

All recruited subjects were invited to attend a follow-up visit by a dedicated psychiatrist 4 weeks after the initial endoscopy. Anti-secretory agents, prokinetics and eradication therapy for Helicobacter pylori were withheld prior to the study follow-up but antacids were allowed as on-demand basis. Severity of dyspeptic symptoms in the past one week was recorded by a self-administrated structured questionnaire at the same time with a adjectival scale of 0 to 3 ( 0 - absence, 1 - mild severity without affecting daily activities, 2 - moderate severity with occasional interference with daily activities, 3 - severe and significantly interfere with most daily activities) on eight different dyspepsia symptoms - epigastric pain, bloating, postprandial fullness, early satiation, nausea, vomiting, belching and epigastric burning. ${ }^{16}$ The overall severity of FD was reflected by the number of severe dyspeptic symptoms (as determined by a score of at least 2) per patient. Presence of irritable bowel symptoms as defined by Rome III criteria was documented, ${ }^{15}$ together with other background information like socio-demographics, alcohol and smoking history, sexual abuse history, and personal past and family histories of psychiatric illness.

The subjects also completed a set of Chinese version of questionnaires, including background information (e.g., education level, economic status, employment, number of sick leaves), SF-36 and GHQ-12 at the visit. ${ }^{17,18}$ Modified Version 2.0 of 
SF-36 was used to determine HRQoL among our recruited subjects. Its Mental Component Summary (MCS) and Physical Component Summary (PCS) scores were calculated based on a cross-sectional study of 2,410 randomly selected Chinese in Hong Kong conducted by the Lam group (Appendix 1). ${ }^{17}$ GHQ-12 was a shorter 12-item version of General Health Questionnaire, which originally consisted of 60 items with four response categories in each item indicating the severity of psychological distress experienced in past four weeks. ${ }^{19}$ It was chosen in this study because it was easy to administer and commonly used worldwide, ${ }^{6}$ and its psychometric properties have been validated in some Chinese outpatient groups. ${ }^{18,20,21}$ Our study used the standard scoring method, in which each response categories scored 0, 0, 1 and 1 respectively. ${ }^{22}$ The summation of scores from all the twelve questions gave a final result ranging from 0 to 12 .

After completion of questionnaire set, a psychiatrist who was trained on the administration of the Chinese-bilingual version of SCID and blinded to the information obtained, conducted the SCID interview. SCID was a semi-structured face-to-face interview for making reliable diagnoses according to DSM-IV. ${ }^{23}$ It has been translated into more than 10 other languages throughout the world, and it is the most widely used psychiatric research tools internationally. SCID-Axis I, patient version (SCID-I/P) consisted of 10 modules covering mood episodes, mood disorders, psychotic symptoms, psychotic disorders, substance use disorders, anxiety disorders, somatoform disorders, eating disorders, adjustment disorders and the last optional module for others. This version of SCID-I/P has been validated in Chinese (Hong Kong) population with good test-retest, intra-rater and inter-rater reliabilities. $^{24-26}$

All data was processed and analyzed by the Statistical Package for Social Science (SPSS Inc., Chicago, IL, USA) version 13.0 for Windows. All continuous variables were presented as mean \pm standard error (SE) and all ordinal variables were presented as median and interquartile range (IQR) unless indicated otherwise. Mann-Whitney $U$ test was used for comparing continuous and ordinal variables. p-value less than 0.05 was considered as statistically significant (two-sided test). A receiver operator characteristic (ROC) curve was plotted for analyzing the sensitivity and specificity of GHQ-12 with reference to the gold standard (i.e., SCID). An area under curve (AUC) of 1.0 indicated a perfect test, while 0.5 or below meant that the test is no better than guessing. In order to confirm caseness (i.e., any Axis I psychiatric disorders), an optimal cut-off score of GHQ-12 with at least $90 \%$ specificity would be determined. The corresponding sensitivity, specificity, positive predictive value (PPV), and negative predictive value (NPV) were calculated as well. Pearson correlation coefficients were calculated to measure how the GHQ-12 score related with HRQoL. Univariate and multivariate linear regression analyses were also adopted to identify the independent risk factors for poorer HRQoL as indicated by lower SF-36 scores.

Based on the results of GHQ-12 in general population, we anticipate that a cut-off score of $\geq 3$ would give rise to a sensitivity of $70 \%$ and specificity of $90 \%$. If the desired precision for specificity is $10 \%$ and prevalence of psychiatric disorders is $33 \%$ among FD patients, ${ }^{1}$ the number of patients needed in our study would be 53 for a confidence interval (CI) of $95 \%$. $^{27}$

\section{Results}

\section{Socio-demographic background}

From September 2007 to February 2008, a total of 260 patients with dyspepsia as the dominant symptoms were screened. Amongst these patients, 108 fulfilled the inclusion criteria, while the others did not have symptom severity diagnostic for FD according to Rome III criteria. Fifty three patients were excluded with following reasons: 36 co-morbid medical problems (16 having hypertension, 11 having diabetes mellitus, 5 having chronic hepatitis B, 2 having gouty arthritis and 2 having menorrhagia), 16 concomitant gastroesophageal reflux disease (GERD), and 1 refusal. As a result, 55 patients were recruited for this study. All patients attended the scheduled follow up and none of them had dyspeptic symptom resolution at study follow-up.

All baseline socio-demographic data were presented in Table 1. Fifteen subjects were male (27.3\%), and the overall mean age was $38.7 \pm 1.5$ years old (range 18-67). Thirty six subjects (65.5\%) were married and 26 subjects $(47.3 \%)$ were educated up to high school or above. Thirty seven subjects (67.3\%) were employed. Seven subjects were active smoker, and none had current drinking habit, although two subjects were ex-drinkers in the past.

\section{Background gastrointestinal symptoms and psychia- tric characteristics}

Concerning the subtyping of our FD patients, 35 patients fulfilled postprandial distress syndrome, 8 fulfilled epigastric pain syndrome, and 12 fulfilled both subtypes. The mean duration of dyspeptic symptoms was $9.4 \pm 1.4$ years. $25.5 \%$ of our patients were Helicobacter pylori carrier, and $23.6 \%$ had co-morbid irritable 
Table 1. Socio-demographic Characteristics

\begin{tabular}{lc}
\hline & Total (n=55) \\
\hline Sex (Male, $\%)$ & $15,27.3 \%$ \\
Age (mean \pm SE) (yr) & $38.7 \pm 1.5$ \\
Marital status (n, \%) & \\
Married & $36,65.5 \%$ \\
Not married & $19,34.5 \%$ \\
Dependent children (n, \%) & \\
None & $30,54.5 \%$ \\
Yes & $15,45.5 \%$ \\
1 & $11,20 \%$ \\
2 & $10,18.2 \%$ \\
3 or more & $4,7.3 \%$ \\
Educational level (n, $\%)$ & \\
Secondary 3 or below & $29,52.7 \%$ \\
Above secondary 3 & $26,47.3 \%$ \\
$\quad$ High school & $11,20 \%$ \\
Tertiary & $12,21.8 \%$ \\
$\quad$ Post-graduate & $3,5.5 \%$ \\
Type of accommodation (n, $\%)$ & \\
Public housing & $25,45.5 \%$ \\
Non-public housing & $30,54.5 \%$ \\
Rented & $9,16.4 \%$ \\
Self owned & $21,38.2 \%$ \\
Institutional & $0,0 \%$ \\
Number of household members $(\mathrm{n}, \%)^{\mathrm{a}}$ & \\
1 & $0,0 \%$ \\
2 & $11,20 \%$ \\
3 & $17,30.9 \%$ \\
5 & $15,27.3 \%$ \\
6 or above & $7,12.7 \%$ \\
& $5,9.1 \%$ \\
\hline
\end{tabular}

bowel syndrome according to Rome III criteria. The median total dyspeptic symptom severity score was 7 . Each patient had a median of 2 severe dyspeptic symptoms that were scored $\geq 2$. For the psychiatric background, 5 participants reported known family history of psychiatric illness in their first or second degree relatives. Only 3 participants $(5.5 \%)$ received past psychiatric intervention, of which only one was still on treatment at the time of study. One participant reported past history of sexual abuse (Table 1).

\section{Structured clinical interview for DSM-IV axis I disorders - current diagnoses}

By using the Chinese-bilingual version of SCID-I/P, the prevalence of any current Axis I psychiatric diagnoses under DSM-IV criteria was $49.1 \%$, but only one patient (3.7\%) was receiving active psychiatric treatment (Table 2 ). The most common group of psychiatric disorders was anxiety disorders (38.3\%), fol-
Table 1. Continued

\begin{tabular}{|c|c|}
\hline & Total $(n=55)$ \\
\hline $\begin{array}{l}\text { Living area per household member } \\
\text { (square feet } \pm \mathrm{SE} \text { ) }\end{array}$ & $159.0 \pm 12.9$ \\
\hline $\begin{array}{l}\text { Income per household member } \\
(\mathrm{HK} \$ \pm \mathrm{SE})\end{array}$ & $6,307.3 \pm 670.3$ \\
\hline \multicolumn{2}{|l|}{ Employment status (n, \%) } \\
\hline Employed & $37,67.3 \%$ \\
\hline Professional/managerial/intermediate & $4,7.3 \%$ \\
\hline Skilled/clerical & $15,27.3 \%$ \\
\hline Semi-skilled/manual & $5,9.1 \%$ \\
\hline Unskilled & $13,23.6 \%$ \\
\hline Not employed & $18,32.7 \%$ \\
\hline Housewife & $10,18.2 \%$ \\
\hline Retired & $1,1.8 \%$ \\
\hline Other economically inactive & $4,7.3 \%$ \\
\hline $\begin{array}{l}\text { Days of paid working in recent } \\
\text { One month (mean } \pm \mathrm{SE})\end{array}$ & $15.2 \pm 1.5$ \\
\hline $\begin{array}{c}\text { Days of sick leave in recent } \\
\text { One month }(\text { mean } \pm \mathrm{SE})\end{array}$ & $0.8 \pm 0.2$ \\
\hline \multicolumn{2}{|l|}{ Smoking habit $(\mathrm{n}, \%)$} \\
\hline Active smoker (daily smoking) & $7,12.7 \%$ \\
\hline \multicolumn{2}{|l|}{ Drinking habit $(\mathrm{n}, \%)$} \\
\hline $\begin{array}{l}\text { Social drinker (moderate alcohol } \\
\text { consumption in socially acceptable } \\
\text { situation, and never met the criteria of } \\
\text { "alcohol abuse" under DSM-IV) }\end{array}$ & $26,47.3 \%$ \\
\hline Never drink & $29,52.7 \%$ \\
\hline Known to psychiatric service (n, \%) & $3,5.5 \%$ \\
\hline Family history of psychiatric illness (n, \%) & $5,9.1 \%$ \\
\hline History of sexual abuse (n, \%) & $1,1.8 \%$ \\
\hline
\end{tabular}

${ }^{a}$ Household member including our study subject.

SE, standard error; DSM-IV, diagnostic and statistical manual of mental disorders, 4th edition.

lowed by mood disorders (16.4\%). After excluding subjects with FD symptoms as the only symptom for the somatoform disorders, there remained $10.8 \%$ having somatization disorder. Last but not least, there were one case of adjustment disorder and one substance abuse.

\section{Structured clinical interview for DSM-IV axis I disorders - past diagnoses}

Seven patients had past depressive episode(s), one patient had past panic disorder, two patients had past post-traumatic stress disorder, and two patients had past alcohol dependence syndrome.

\section{Performance of GHQ-12 for psychiatric disorders}

Our subjects were classified into two groups: 
Table 2. Results of SCID - Current Psychiatric Morbidities

\begin{tabular}{ll}
\hline \multicolumn{1}{c}{ Diagnosis $^{\mathrm{a}}$} & \multicolumn{1}{c}{$\mathrm{n}(\%)$} \\
\hline No axis I diagnosis & $28(50.9 \%)$ \\
Any axis I diagnosis & $27(49.1 \%)$ \\
More than one axis I diagnosis & $11(20 \%)$ \\
Anxiety disorders & $21(38.2 \%)$ \\
Generalized anxiety disorder & $14(25.5 \%)$ \\
Mixed anxiety depressive disorder & $6(10.9 \%)$ \\
Other anxiety disorder NOS & $1(1.8 \%)$ \\
Specific phobia & $6(10.9 \%)$ \\
Social phobia & $1(1.8 \%)$ \\
Panic disorder & $0(0 \%)$ \\
Agoraphobia without panic disorder & $0(0 \%)$ \\
Obsessive compulsive disorder & $0(0 \%)$ \\
Post traumatic stress disorder & $0(0 \%)$ \\
Mood disorders & $9(16.4 \%)$ \\
Dysthmia & $7(12.7 \%)$ \\
Major depressive episode & $2(3.6 \%)$ \\
Bipolar disorder & $0(0 \%)$ \\
Somatoform disorders & $6(10.9 \%)$ \\
Somatization disorder & $6(10.9 \%)$ \\
Hypochondriasis & $0(0 \%)$ \\
Schizophrenia \& other psychotic disorders & $0(0 \%)$ \\
Eating disorders & $0(0 \%)$ \\
Adjustment disorders & $1(1.8 \%)$ \\
Substance abuse & $1(1.8 \%)$ \\
\hline
\end{tabular}

${ }^{\mathrm{a} A l l}$ psychiatric diagnoses were made after SCID, ${ }^{\mathrm{b}}$ Symptoms of functional dyspepsia were excluded for somatoform disorders.

1) Subjects with any current Axis I Psychiatric Disorders by SCID-I/P $(n=27)$

2) Subjects without current Axis I Psychiatric Disorders by $\mathrm{SCID}-\mathrm{I} / \mathrm{P}(\mathrm{n}=28)$

The GHQ-12 score for patients with psychiatric disorders was significantly higher than those without $[$ Median score $=4$ $(\mathrm{IQR}=1-6)$ versus $1(\mathrm{IQR}=0-2), \mathrm{p}<0.001]$ (Table 3$)$. The ROC curve was plotted as shown in Fig. 1, and the AUC for GHQ-12 was 0.825 (95\% CI $=0.698-0.914)$.

An optimal cut-off score of $\geq 3$ was found for at least $90 \%$ specificity. Then the relevant sensitivity, specificity, PPV and NPV were 63.0\% (95\% CI $=42.4-80.6 \%), 92.9 \%$ (95\% CI $=$ $76.5-98.9 \%), 89.5 \%$ (95\% CI $=66.0-98.5 \%)$, and $72.2 \%(95 \%$ $\mathrm{CI}=54.8-85.8 \%)$ respectively. SF-36 scores were also compared according to this cut-off of GHQ-12. For score $\geq 3$, the mean PCS and MCS were $36.8 \pm 2.3$ and $35.6 \pm 2.5$, which were significantly lower than score $<3(\mathrm{PCS}=44.1 \pm 1.3, \mathrm{p}=$ $0.005 ; \mathrm{MCS}=47.1 \pm 1.4, \mathrm{p}<0.001)$ respectively.

\section{Dyspeptic symptom severity}

There was no statistically significant difference in each dyspeptic symptom score between patients with and without psychiatric disorders. The overall severity of FD was also similar in both groups as reflected by no significant difference in the number of severe dyspeptic symptoms (score $\geq 2$ ) per patient (Table $3)$.

\section{Health-related quality of life}

Results of SF-36 were shown in Table 3. The overall mean MCS and PCS scores were $43.9 \pm 1.5$ and $40.7 \pm 1.2$ respectively. For patients with psychiatric comorbidities, multiple domains of SF-36 including MCS, general health, vitality and mental health were significantly lower than those without psychiatric illness. However, there was no statistically significant difference in PCS between the two groups $(39.3 \pm 2.1$ versus $43.7 \pm$ $1.3, \mathrm{p}=0.072)$. Furthermore, HRQoL was found to be related with GHQ-12 score significantly [Pearson correlation coefficient: -0.486 (versus PCS), $\mathrm{p}<0.001 ;-0.613$ (versus MCS), $\mathrm{p}<0.001]$.

\section{Risk factors for poor health-related quality of life}

By univariate linear regression analysis, 6 potential factors ("current psychiatric disorder", "family history of psychiatric illness", "number of severe dyspeptic symptoms per patient", "co-morbid irritable bowel syndrome according to Rome III criteri", "marital status", and "age", all p-value < 0.1) have been found to be associated with poorer HRQoL in FD patients, as indicated by either lower MCS or PCS scores. Nevertheless, in multivariate analysis, only "current psychiatric disorder" (Beta = $-0.396, p=0.002)$ and "family history of psychiatric illness" (Beta $=-0.299, \mathrm{p}=0.015)$ were the independent risk factors for lower MCS. On the other hand, higher "number of severe dyspeptic symptoms per patient" was the only independent risk factor for lower PCS (Beta $=-0.332, \mathrm{p}=0.027)$.

\section{Discussion}

Using a gold standard diagnostic method, we have shown that psychiatric disorders were highly prevalent among FD patients newly referred from primary care setting, affecting $49.1 \%$ of our patients. Anxiety disorders (38.2\%), depressive disorders $(16.4 \%)$ and somatization disorders $(10.8 \%)$ were the most common diagnoses by SCID. Furthermore, we have found that 
Table 3. Results of Self-administrated Questionnaires at Post-esophagogastroduodenoscopy Follow-up

\begin{tabular}{|c|c|c|c|c|}
\hline & \multicolumn{4}{|c|}{ Questionnaires' Scores } \\
\hline & \multirow{2}{*}{ All } & \multicolumn{3}{|c|}{ Current Psychiatric Disorder(s) } \\
\hline & & Yes & No & $p$-value ${ }^{a}$ \\
\hline \multicolumn{5}{|l|}{ Dyspeptic symptom score (Total) } \\
\hline Epigastric pain ${ }^{\mathrm{b}}$ & $1,1-2$ & $1,1-2$ & $1,1-2$ & 0.321 \\
\hline Belching ${ }^{\mathrm{b}}$ & $1,1-2$ & $1,1-2$ & $1,0-2$ & 0.305 \\
\hline Epigastric burning $^{\mathrm{b}}$ & $0,0-1$ & $1,0-1$ & $0,0-1$ & 0.131 \\
\hline Bloating $^{\mathrm{b}}$ & $1,1-2$ & $1,1-2$ & $1,0-2$ & 0.071 \\
\hline Post-prandial fullness ${ }^{\mathrm{b}}$ & $1,1-2$ & $1,1-2$ & $1,1-2$ & 0.224 \\
\hline Early satiety ${ }^{\mathrm{b}}$ & $1,0-1$ & $1,0-2$ & $1,0-1$ & 0.329 \\
\hline Nausea $^{\mathrm{b}}$ & $0,0-1$ & $1,0-1$ & $0,0-1$ & 0.246 \\
\hline Vomiting $^{\mathrm{b}}$ & $0,0-0$ & $0,0-1$ & $0,0-0$ & 0.172 \\
\hline $\begin{array}{l}\text { No. of severe dyspeptic symptoms } \\
\text { (score } \geq 2 \text { ) per patient }^{\mathrm{b}}\end{array}$ & $2,0-4$ & $2,0-4$ & $1,0-3$ & 0.251 \\
\hline GHQ-12 ${ }^{c}$ score $^{b}$ & $2,1-4$ & $4,1-6$ & $1,0-2$ & $<0.001$ \\
\hline \multicolumn{5}{|l|}{ HK SF- $36^{d}$ score $^{e}$} \\
\hline Physical component summary & $40.7 \pm 1.2$ & $39.3 \pm 2.1$ & $43.7 \pm 1.3$ & 0.072 \\
\hline Mental component summary & $43.9 \pm 1.5$ & $37.8 \pm 2.0$ & $48.2 \pm 1.6$ & $<0.001$ \\
\hline Physical functioning & $46.2 \pm 1.6$ & $43.1 \pm 2.6$ & $49.5 \pm 1.6$ & 0.055 \\
\hline Role physical & $44.7 \pm 1.1$ & $42.7 \pm 1.7$ & $46.7 \pm 1.3$ & 0.108 \\
\hline Bodily pain & $34.5 \pm 1.2$ & $33.9 \pm 2.0$ & $35.1 \pm 1.5$ & 0.664 \\
\hline General health & $44.0 \pm 1.2$ & $40.5 \pm 1.7$ & $47.4 \pm 1.4$ & 0.010 \\
\hline Vitality & $44.1 \pm 1.5$ & $38.9 \pm 2.1$ & $48.9 \pm 1.5$ & $<0.001$ \\
\hline Social functioning & $37.2 \pm 1.9$ & $33.8 \pm 3.0$ & $40.5 \pm 2.3$ & 0.141 \\
\hline Role emotional & $48.4 \pm 1.0$ & $46.6 \pm 1.4$ & $50.2 \pm 1.2$ & 0.059 \\
\hline Mental health & $41.6 \pm 1.5$ & $35.2 \pm 1.7$ & $48.1 \pm 1.5$ & $<0.001$ \\
\hline
\end{tabular}

${ }^{\mathrm{a}}$ All comparisons between patients with and without psychiatric illness were done by Mann Whitney $\mathrm{U}$ test, ${ }^{\mathrm{b}}$ Presented as median, interquartile range, ${ }^{\mathrm{c}} \mathrm{GHQ}-12=12$-item General Health Questionnaire, ${ }^{\mathrm{d}} \mathrm{HK}$ SF-36 = Chinese (Hong Kong) version of 36-item short-form health survey, Presented as mean \pm standard error.

GHQ-12 was a reliable screening instrument for psychiatric disorders in patients with FD.

To the author's knowledge, this is the first study validating a self-rated questionnaire for screening psychiatric disorders in FD patients against a gold standard psychiatrist-conducted interview. With an accurate and simple screening tool, early detection and treatment of any underlying psychiatric disorders are made possible. Cross validation between GHQ-12 and SF-36 scores also shows that GHQ-12 is a useful tool to estimate the morbidity of FD patients. In other words, GHQ-12 helps us to identify newly referred FD patients from primary care who warrant full psychiatric assessment.

It is not uncommon that patients with GERD also report dyspepsia-like symptoms. Because of the different pathophysiology, presence of typical reflux symptoms more than once a week on top of dyspepsia should lead to a provisional diagnosis of GERD instead of FD. ${ }^{15}$ On the other hand, literature suggests that at least subsets of FD and IBS represent different manifes- tations of a single entity, and there exists considerable symptom overlap. $^{28,29}$ Therefore we excluded all patients with GERD but not IBS during our subject recruitment. More importantly, co-morbid medical illness is a common occurrence in such a referral setting and it is a well-known risk factor for psychiatric morbidity. ${ }^{30}$ To tackle with this problem, significant medical illnesses were excluded from our subject recruitment. Moreover, only newly referred out-patients were recruited in current study to avoid selective sampling of a more severe spectrum of FD. For instance, the SF-36 scores in our subjects were comparable to a Spanish multi-centre primary care study on FD patients. ${ }^{8}$

In our study, ROC curve analysis showed that performance of GHQ-12 was good for detecting psychiatric illness in FD patients, with an AUC of 0.825 (95\% CI $=0.698-0.914)$. The cut-off value would be aimed at earlier recognition and treatment for otherwise undiagnosed psychiatric disorders. ${ }^{6}$ On the other hand, the cost of false positives has to be weighed against the cost of missing psychiatric conditions. Having considered all the 


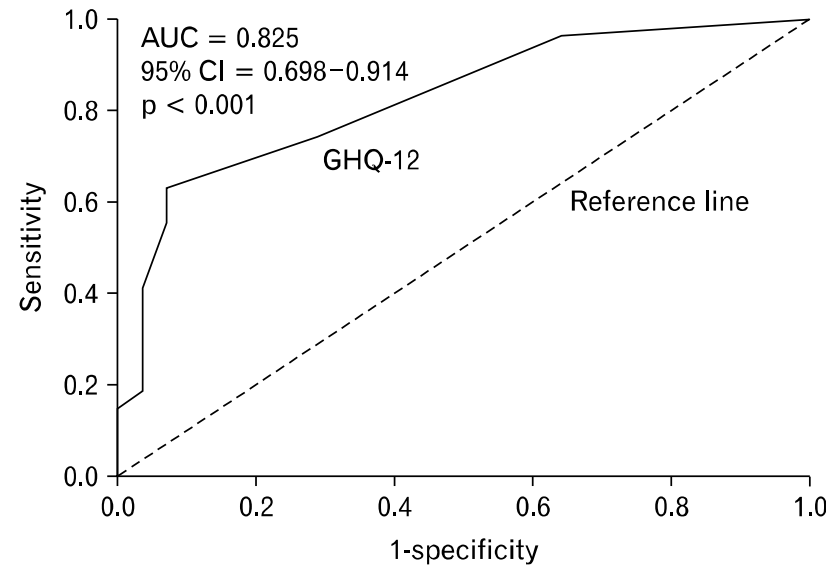

Figure 1. ROC curve of GHQ-12 to detect psychiatric comorbidities.

above factors, a cut-off score of $\geq 3$ for deciding psychiatric referral was chosen to achieve optimal sensitivity and specificity.

Both MCS and PCS of SF-36 were low in FD patients, as compared to the standardized mean scores (i.e., 50) in Hong Kong general population (Table 3). ${ }^{17}$ In multivariate analysis, the "number of severe dyspeptic symptoms per patient" was the independent risk factor for lower PCS, while "current psychiatric disorder" and "family history of mental illness" were independently associated with lower MCS. On the other hand, there existed no significant difference in PCS and dyspeptic symptom severity scores between patients with and without psychiatric co-morbidities. Translating into clinical implications, although HRQoL were globally impaired in FD patients, the mental component is more affected by the presence of psychiatric illness either in the dyspeptic patient or his/her family members, while the physical component is more affected by dyspeptic symptom severity. This finding is consistent with the conclusion from several studies that only some of the impairment in functional gastrointestinal disorder (FGID) can be accounted by gastrointestinal symptoms. ${ }^{10,12}$

In our cohort, only one patient was receiving active psychiatric care during recruitment, and two patients had past history of psychiatric treatment. This illustrated the poor awareness of psychiatric disorders and low rate of psychiatric intervention received in our study patients, which further supports the need for a reliable and simple screening tool in non-psychiatrist setting.

There were limitations in this study. Firstly, our sample size was relatively small which was primarily limited by the workload required for lengthy psychiatrist-conducted interview. Despite early screening and assessment of our patients newly referred from primary care setting, they might not represent primary care patients who might have milder symptoms and lower degree of psychological comorbidity. In order to estimate the prevalence of psychiatric disorders and usefulness of GHQ-12 in community, a large-scale study at primary care setting is warranted by using GHQ-12 with our determined cut-off value. Furthermore, other psychosocial covariates such as personality traits, coping strategies and life events were not fully evaluated here. Although it has been suspected that such factors may interfere with health-care seeking behaviour, the impact of these factors among patients with FGID is still highly controversial. ${ }^{3,31-33}$ Last but not the least, the severity of psychiatric disorders was not quantified and recorded systemically in our study. However, we believe that $\mathrm{HRQ} \mathrm{LL}$, as measured by SF-36, is a reliable reflection of the severity and impairment of psychiatric illness.

In conclusion, psychiatric comorbidities are common amongst $\mathrm{FD}$, yet only a small proportion is recognized and properly treated. GHQ-12 has good performance in screening psychiatric disorders in FD patients, which is important as concomitant psychiatric illnesses adversely affect the HRQoL of these patients, especially the mental component. GHQ-12 serves as a useful tool that facilitates early recognition of psychiatric disorders in non-psychiatrist clinical setting for FD patients.

\section{References}

1. Haug TT, Svebak S, Wilhelmsen I, Berstad A, Ursin H. Psychological factors and somatic symptoms in functional dyspepsia. A comparison with duodenal ulcer and health controls. J Psychosom Res 1994;38:281-291.

2. Talley NJ, Fung LH, Gilligan IJ, McNeil D, Piper DW. Association of anxiety, neuroticism, and depression with dyspepsia of unknown cause. A case-control study. Gastroenterology 1986;90: 886-892.

3. Talley NJ, Phillips SF, Bruce B, Twomey CK, Zinsmeister AR, Melton LJ 3rd. Relation among personality and symptoms in nonulcer dyspepsia and the irritable bowel syndrome. Gastroenterology 1990;99:327-333.

4. Langeluddecke P, Goulston K, Tennant C. Psychological factors in dyspepsia of unknown cause: a comparison with peptic ulcer disease. J Psychosom Res 1990; 34: 215-222.

5. Werneke U, Goldberg DP, Yalcin I, Ustün BT. The stability of the factor structure of the General Health Questionnaire. Psychol Med 2000;30:823-829.

6. Golderg DP, Gater R, Sartorius N, et al. The validity of two versions of GHQ in the WHO study of mental illness in general health care. Psychol Med 1997;27:191-197.

7. Talley NJ, Weaver AL, Zinsmeister AR. Impact of functional dyspepsia on quality of life. Dig Dis Sci 1995;40:584-589.

8. Enck P, Dubois D, Marquis P. Quality of life in patients with upper 
gastrointestinal symptoms: results from the domestic/international gastroenterology surveillance study (DIGEST). Scand J Gastroenterol 1999;231:48-54.

9. Monés J, Adan A, Segú JL, López JS, Artés M, Guerrero T. Quality of life in functional dyspepsia. Dig Dis Sci 2002;47:20-26.

10. Halder SL, Locke GR 3rd, Talley NJ, Fett SL, Zinsmeister AR, Melton LJ 3rd. Impact of functional gastrointestinal disorders on health-related quality of life: a population-based case-control study. Aliment Pharmacol Ther 2004;19:233-242.

11. El-Serag HB, Talley NJ. Health-related quality of life in functional dyspepsia. Aliment Pharmacol Ther 2003;18 387-393.

12. Talley NJ, Locke GR 3rd, Lahr BD, et al. Functional dyspepsia, delayed gastric emptying, and impaired quality of life. Gut 2006;55: 933-939.

13. Functional Dyspepsia Module. Rome Foundation, Inc. Tampa, Florida, USA. Available from: http://www.romecriteria.org/pdfs/ DyspepMode.pdf (Accessed 6th August, 2009)

14. Chiu HF, Lam LC, Chi I, et al. Prevalence of dementia in Chinese elderly in Hong Kong. Neurology 1998; 50:1002-1009.

15. Tack J, Talley NJ, Camilleri M, et al. Functional gastroduodenal disorders. Gastroenterology 2006;130:1466-1479.

16. Tack J, Piessevaux H, Coulie B, Caenepeel P, Janssens J. Role of impaired gastric accommodation to a meal in functional dyspepsia. Gastroenterology 1998;115:1346-1352.

17. Lam CL, Tse EY, Gandek B, Fong DY. The SF-36 summary scales were valid, reliable and equivalent in a Chinese population. J Clin Epidemiol 2005;58:815-822.

18. Pan PC, Goldberg DP. A comparison of the validity of GHQ-12 and CHQ-12 in Chinese primary care patients in Manchester. Psychol Med 1990;20:931-940.

19. General Health Questionnaire. StatPac, Inc. Bloomington, Minnesota, USA. Available from: http://www.webpoll.org/psych/ GHQ12.htm (Accessed 6th August, 2009)

20. Ip WY, Martin CR. Psychometric properties of the 12-item General Heath Questionnaire (GHQ-12) in Chinese women during pregnancy and in the postnatal period. Psycho Health Med 2006; 11:60-69.

21. Lee DT, Yip AS, Chiu HF, Chung TK. Screening for postnatal depression using the double-test strategy. Psychosom Med 2000;62: 258-263.

22. Piccinelli M, Bisoffi G, Bon MG, Cunico L, Tansella M. Validity and test-retest reliability of the Italian version of the 12-item General Health Questionnaire in general practice: a comparison between three scoring methods. Compr Psychiatry 1993;34:198-205.

23. First MB, Gibbon M, Spitzer RL, Williams JB. Structured Clinical Interview for Axis I DSM-IV Disorders-Patient Edition (SCID-I/P, Version 2.0, February 1996 Final Version). Biometrics Research Department, New York State Psychiatric Institute.

24. So E, Kam I, Leung CM, Chung D, Liu Z, Fong S. The Chinese-bilingual SCID-I/P project: stage 1-reliability for mood disorders and schizophrenia. Hong Kong J Psychiatry 2003;13:7-18.

25. So E, Kam I, Leung CM, Pang A, Lam L. The Chinese-bilingual SCID-I/P project: stage 2 - reliability for anxiety disorders, adjustment disorders, and "no diagnosis". Hong Kong J Psychiatry 2003;13:19-25.

26. So E, Kam I, Lam L. The Chinese-bilingual SCID-I/P project: stage 3 - multi-site inter-rater reliability. Hong Kong J Psychiatry 2005;14:19-25.

27. Jones SR, Carley S, Harrison M. An introduction to power and sample size estimation. Emerg Med J 2003;20:453-458.

28. Cremonini F, Talley NJ. Review article: the overlap between functional dyspepsia and irritable bowel syndrome - a tale of one or two disorders? Aliment Pharmacol Ther 2004;20(suppl 7):40-49.

29. Riedl A, Schmidtmann M, Stengel A, et al. Somatic comorbidities of irritable bowel syndrome: a systemic analysis. J Psychosom Res 2008;64:573-582.

30. Wells KB, Golding JM, Burnam MA. Psychiatric disorder in a simple of the general population with and without chronic medical conditions. Am J Psychiatry 1988;145:976-981.

31. Haug TT, Wilhelmsen I, Berstad A, Ursin H. Life events and stress in patients with functional dyspepsia compared with patients with duodenal ulcer and healthy controls. Scand J Gastroenterol 1995;30: 524-530.

32. Lee S, Park M, Choi S, Nah Y, Abbey SE, Rodin G. Stress, coping, and depression in non-ulcer dyspepsia patients. J Psychosom Res 2000;49:93-99.

33. Koloski NA, Boyce PM, Talley NJ. Somatization an independent psychosocial risk factor for irritable bowel syndrome but not dyspepsia: a population-based study. Eur J Gastroenterol Hepatol 2006;18: 1101-1109.

\section{Acronyms}

$\begin{array}{ll}\text { AUC } & \text { Area Under Curve } \\ \text { CI } & \text { Confidence Interval } \\ \text { DSM-IV } & \begin{array}{l}\text { Diagnostic and Statistical Manual of } \\ \text { Mental Disorders, } 4^{\text {th }} \text { Edition }\end{array} \\ \text { FD } & \text { Functional Dyspepsia } \\ \text { FGID } & \text { Functional Gastrointestinal Disorder } \\ \text { GERD } & \text { Gastroesophageal Reflux Disease } \\ \text { GHQ-12 } & \text { 12-item General Health Questionnaire } \\ \text { HRQDL } & \text { Health-related Quality Of Life } \\ \text { MCS } & \text { Mental Component Summary } \\ \text { MMSE } & \text { Mini-mental State Exam } \\ \text { NPV } & \text { Negative Predicitive Value } \\ \text { PCS } & \text { Physical Component Summary } \\ \text { PPV } & \text { Positive Predictive Value } \\ \text { ROC } & \text { Receiver Operating Characteristic } \\ \text { SCID } & \text { Structured Clinical Interview for DSM-IV - } \\ & \text { Axis I Disorders } \\ \text { SCID-I/P } & \text { SCID - Axis I, Patient Version } \\ \text { SE } & \text { Standard Error } \\ \text { SF-36 } & \text { 36-item Short-form Health Survey }\end{array}$


SF_Z $=($ SF-91.19295) $/ 16.56710$

RE_Z $=($ RE-71.65975) $/ 38.36354$

Appendix

MH_Z $=(\mathrm{MH}-72.87506) / 16.56739$

Appendix 1. Hong Kong-specific Scoring Algorithms for $\mathrm{SF}-36$

Scoring algorithm for the Hong Kong-specific SF-36 PCS and MCS scales: ${ }^{16}$

PF_Z $=($ PF-91.82573) $/ 12.88527$

$\mathrm{RP} Z=(\mathrm{RP}-82.42739) / 30.97154$

BP Z $=($ BP-83.97801)/21.89251

GH_Z $=(\mathrm{GH}-55.97759) / 20.17986$

VT_Z $=($ VT-60.27178) / 18.64714
HK-specific SF-36 MCS Score $=(-\mathrm{PF} Z \mathrm{Z} \times 0.22743+$ RP_Z $\times 0.01327-B P \_Z \times 0.09483-G H \_Z \times 0.05122$ + VT_Z $\times 0.25123+\mathrm{SF}_{-} \mathrm{Z} \times 0.33064+\mathrm{RE} \_\mathrm{Z} \times$ $0.44834+$ MH_Z $\times 0.41167) \times 10+50$

HK-specific SF-36 PCS Score $=\left(P F \_Z \times 0.46095+\right.$ $\mathrm{RP} Z \mathrm{Z} \times 0.27474+\mathrm{BP}_{-} \mathrm{Z} \times 0.35475+\mathrm{GH} Z \mathrm{Z} \times$ $0.32470+$ VT_Z $\times 0.03257-\mathrm{SF} Z \mathrm{Z} \times 0.07846-\mathrm{RE} \_Z$ $\times 0.19399-$ MH_Z $\times 0.12198) \times 10+50$ 